\title{
Search for Ultra-relativistic Magnetic Monopoles with the Pierre Auger Observatory
}

\author{
Toshihiro Fujii $^{\ngtr a}$ for the Pierre Auger Collaboration ${ }^{b}$ \\ ${ }^{a}$ Kavli Institute for Cosmological Physics, University of Chicago, Chicago, 60637 Illinois, USA \\ ${ }^{b}$ Observatorio Pierre Auger, Av. San Martín Norte 304, 5613 Malargüe, Argentina \\ E-mail: auger_spokespersons@fnal.gov \\ Full author list: http://Www.auger.org/archive/authors_2015_06.htm
}

\begin{abstract}
Ultra-relativistic magnetic monopoles, possibly a relic of phase transitions in the early universe, would deposit an amount of energy comparable to UHECRs in their passage through the atmosphere, producing highly distinctive air shower profiles. We have performed a search for ultra-relativistic magnetic monopoles in the sample of air showers with profiles measured by the fluorescence detector of the Pierre Auger Observatory. No candidate was found to satisfy our selection criteria and we establish upper limits on the flux of ultra-relativistic magnetic monopoles - the first from an UHECR detector - improving over previous results by up to an order of magnitude.
\end{abstract}

The 34th International Cosmic Ray Conference,

30 July- 6 August, 2015

The Hague, The Netherlands

\footnotetext{
*Speaker.
} 


\section{Introduction}

In 1931, Dirac showed that the existence of magnetic monopoles would explain the quantization of the electric charge from the relation between the unit of electric charge $e$ and the unit of magnetic charge $g_{M}=N e / 2 \alpha$ [四], where $\alpha$ is the fine-structure constant and $N$ is an integer. In addition, it would bring Maxwell's equations to a complete symmetry. Within the framework of Grand Unified Theories (GUT), supermassive magnetic monopoles $\left(M \approx 10^{26} \mathrm{eV} / \mathrm{c}^{2}\right)$ may be produced in the early Universe as intrinsically stable topological defects at the phase transition corresponding to the spontaneous symmetry breaking of the unified fundamental interactions [[]]. Lower mass monopoles may come from later phase transitions at lower energy scales. These particles may be present in the Universe today as a relic of these transitions. Experimental searches for magnetic monopoles are based on their velocity-dependent interactions with matter, with a wide range of velocities allowed for GUT monopoles. Supermassive GUT magnetic monopoles should be gravitationally bound to the Galaxy (or to the Sun and Earth) with non-relativistic virial velocities [[]]. Lighter magnetic monopoles can reach relativistic velocities through acceleration in coherent domains of the galactic and intergalactic magnetic fields, as well as in the vicinity of astrophysical objects (e.g. neutron stars) [B]. Kinetic energies of the order of $10^{25} \mathrm{eV}$ have been predicted [䧃], which result in ultra-relativistic velocities for intermediate mass monopoles (IMMs, $\left.M \sim 10^{11}-10^{20} \mathrm{eV} / \mathrm{c}^{2}\right)$.

There is a long history of experimental searches for magnetic monopoles with a variety of

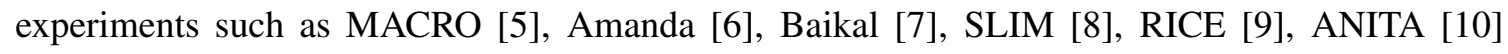
and IceCube [ए]]. The strongest upper limit on the flux of non-relativistic magnetic monopoles $(\beta=v / c<0.5)$ comes from the MACRO experiment at $\approx 1.5 \cdot 10^{-16}\left(\mathrm{~cm}^{2} \mathrm{sr} \mathrm{s}\right)^{-1}(90 \%$ C.L. $)$ [[]]. At relativistic velocities $(\beta \approx 0.9)$, the IceCube Observatory has placed the best limit at $10^{-19}$ $\left(\mathrm{cm}^{2} \mathrm{sr} \mathrm{s}\right)^{-1}$ [Ш]]. The best limit on the flux of ultra-relativistic IMM (Lorentz factor $\gamma \geq 10^{10}$ ) is reported by the ANITA-II experiment at $10^{-19}\left(\mathrm{~cm}^{2} \mathrm{sr} \mathrm{s}\right)^{-1}$ [ए]].

The Pierre Auger Observatory is the largest detector to observe ultra-high energy cosmic rays currently in operation [12] located in the southern hemisphere in central Argentina, just north east of the town of Malargüe $\left(69^{\circ} \mathrm{W}, 35^{\circ} \mathrm{S}, 1400 \mathrm{~m}\right.$ a.s.1.), and covers a ground area of 3,000 $\mathrm{km}^{2}$. The Pierre Auger Observatory consists of a surface detector array (SD) of 1660 individual water-Cherenkov surface detectors [[3] overlooked by a fluorescence detector (FD) of 24 individual fluorescence telescopes grouped in units of 6 at four locations [[4]]. Since the FD steadily observes UHECRs using a huge target volume in the atmosphere with high precision measurements, it would be a suitable detector to search for signals generated from ultra-relativistic IMMs. Therefore, we search for ultra-relativistic IMMs with data collected by the FD between 01.12.2004 and 31.12.2012.

\section{Air Shower Simulations for the IMM Search}

Electromagnetic interactions of magnetic monopoles have been extensively investigated [प15]. The electromagnetic energy loss of a magnetic monopole in air is shown in Figure $\mathbb{W}$ (a) as a function of its Lorentz factor. Collisional energy loss of a magnetic monopole is the dominant contribution for $\gamma \leq 10^{4}$. At higher Lorentz factors, pair production and photonuclear interactions 


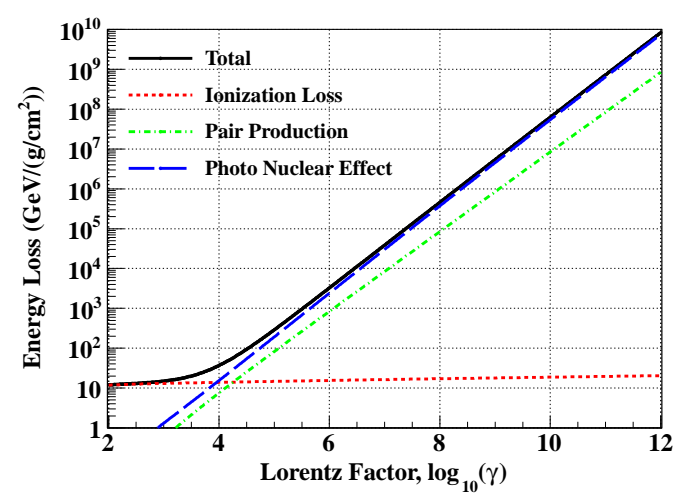

(a) Energy loss

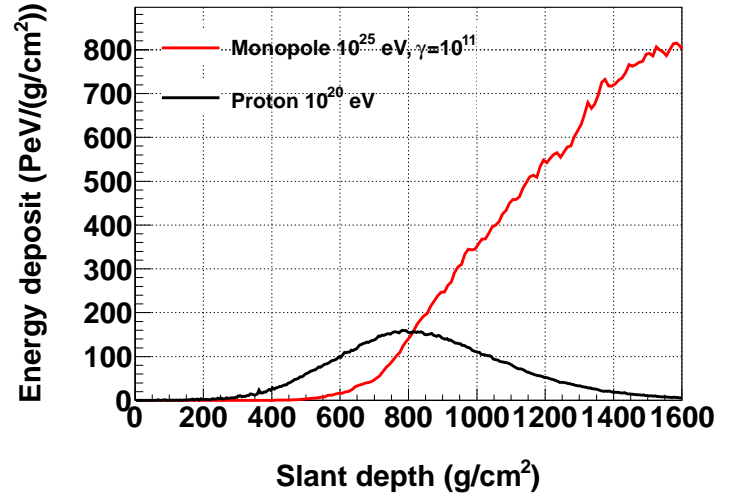

(b) IMM profile

Figure 1: (a) Energy loss of a magnetic monopole in air as a function of its Lorentz factor $\gamma$. (b) Longitudinal profile of the energy deposited by an ultra-relativistic IMM of $E_{\mathrm{mon}}=10^{25} \mathrm{eV}, \gamma=10^{11}$ and zenith angle of $70^{\circ}$ (red solid line). The profile of an UHECR proton shower of energy $10^{20} \mathrm{eV}$ is shown as a black solid line.

become the main cause of energy loss. Bremsstrahlung is highly suppressed by the large monopole mass. An ultra-relativistic IMM would deposit a large amount of energy in its passage through the Earth's atmosphere, comparable to that of an UHECR. For example, an IMM with $\gamma=10^{11}$ loses $\approx 400 \mathrm{PeV} /\left(\mathrm{g} / \mathrm{cm}^{2}\right.$ ) (cf. Figure $\mathrm{W}(\mathrm{a})$ ), which sums up to $\approx 10^{20.5} \mathrm{eV}$ when integrated over an atmospheric depth of $\approx 1000 \mathrm{~g} / \mathrm{cm}^{2}$. This energy will be dissipated by the IMM through production of secondary showers along its path.

The longitudinal profile of the energy deposited by an ultra-relativistic IMM of $E_{\mathrm{mon}}=10^{25} \mathrm{eV}$, $\gamma=10^{11}$ and zenith angle of $70^{\circ}$ is shown in Figure $\mathrm{W(b)}$. When compared with a standard UHECR proton shower of energy $10^{20} \mathrm{eV}$ (black solid line in Figure $\mathrm{W}(\mathrm{b})$ ), the IMM shower presents a much larger energy deposit and deeper development, due to the superposition of many showers uniformly produced by the IMM along its path in the atmosphere. This distinctive feature will be used in our analysis, which is based on the shower development measured by the FD and SD events.

Monte Carlo samples of ultra-relativistic IMM were simulated for Lorentz factors in the range $\gamma=10^{8}-10^{12}$ at a fixed monopole energy $E_{\text {mon }}$ of $10^{25} \mathrm{eV}$. While we used a fixed $E_{\text {mon }}$ in the simulations, the results can be readily applied to a much larger range of monopole energies, since in the ultra-relativistic regime of this search the monopole energy loss does not depend on $E_{\text {mon }}$ but rather on $\gamma$. To estimate the background from UHECRs, we simulated proton showers with energy $E_{\mathrm{p}}$ between $10^{18} \mathrm{eV}$ and $10^{21} \mathrm{eV}$ by three different models, QGSJetII-04, Sibyll 2.1 and Epos-LHC. We used three different models to account for uncertainties in the hadronic interactions. Events were simulated according to an $E_{\mathrm{p}}^{-1}$ energy spectrum, to ensure sufficient Monte Carlo statistics at the highest energy, and then appropriately weighted to reproduce the energy spectrum measured by the Pierre Auger Observatory [ए6]. For both the IMM and UHECR simulations, we used the CORSIKA package [ㅍ] to generate an isotropic distribution of showers above the horizon, and the Auger Offline software [18]] to produce the corresponding FD and SD events. 


\begin{tabular}{lrc}
\hline Event selection criteria & \# events & $(\%)$ \\
\hline Reconstructed events & 376084 & - \\
Zenith angle $<60^{\circ}$ & 360159 & 95.8 \\
Distance from nearest $\mathrm{SD}<1500 \mathrm{~m}$ & 359467 & 99.8 \\
Number of FD pixels $>5$ & 321293 & 89.4 \\
Slant depth interval $>200 \mathrm{~g} / \mathrm{cm}^{2}$ & 205165 & 63.9 \\
Gaps in profile $<20 \%$ & 199625 & 97.3 \\
profile fit $\chi^{2} / \mathrm{ndf}<2.5$ & 197293 & 98.8 \\
$d E /\left.d X\right|_{X \text { up }}>3.0 \mathrm{PeV} /\left(\mathrm{g} / \mathrm{cm}^{2}\right)$ & 6812 & 3.5 \\
$X_{\max }>X_{\text {up }}$ & 352 & 5.2 \\
$X_{\text {up }}>1080 \mathrm{~g} / \mathrm{cm}^{2}$ or $d E /\left.d X\right|_{X \text { up }}>150 \mathrm{PeV} /\left(\mathrm{g} / \mathrm{cm}^{2}\right)$ & 0 & 0.0 \\
\hline
\end{tabular}

Table 1: Event selection criteria and unblinded result. The number of events passing each selection criteria is reported, together with the corresponding fraction.

\section{Event Selection}

Before shower selection, only time periods with a good status of FD telescopes and a high quality calibration of the gains of PMTs are selected using information in Auger databases. Additional cuts are applied to assure good atmospheric conditions due to aerosols and clouds. A further set of selection criteria was applied to ensure good quality showers as summarized in Table $\mathrm{U}$. Those criteria for IMM selection were established from Monte Carlo simulations described in Section $\square$.

The important parameters for the IMM search are the slant depth at the upper field-of-view boundary, $X_{\text {up }}$, and the energy deposited at $X_{\text {up }}, d E /\left.d X\right|_{X \text { up }}$. The requirement $d E /\left.d X\right|_{X \text { up }}>3.0$ $\mathrm{PeV} /\left(\mathrm{g} / \mathrm{cm}^{2}\right)$ is equivalent to an energy threshold of $\approx 10^{18.5} \mathrm{eV}$, where the SD is fully efficient. When $X_{\max }>X_{\text {up }}$ is required, the number of proton backgrounds is drastically reduced and become constrained in a much smaller region, as shown in Figure $\nabla(a)$. On the other hand, the reconstructed $X_{\max }$ will always be outside the FD field of view for ultra-relativistic IMM showers, independently of the shower's $X_{\text {up }}$. This is apparent in Figure $\nabla(b)$, where the correlation of $d E /\left.d X\right|_{X \text { up }}$ with $X_{\text {up }}$ is shown for ultra-relativistic IMM simulated events. The background from UHECRs is almost eliminated by excluding an appropriate region of the $\left(X_{\mathrm{up}}, d E /\left.d X\right|_{X \text { up }}\right)$ plane. We optimized the selection to achieve less than 0.1 background events expected in the data set of this search. The final requirement, $X_{\text {up }}>1080 \mathrm{~g} / \mathrm{cm}^{2}$ or $d E /\left.d X\right|_{X \text { up }}>150 \mathrm{PeV} /\left(\mathrm{g} / \mathrm{cm}^{2}\right)$, is shown in Figure $\square$ as a dashed box, and results in an expected background of 0.07 events in the search period data set.

\section{Data Analysis and Results}

The search for ultra-relativistic IMM was performed following a blind procedure. The selection criteria described in Section [] were optimized using Monte Carlo simulations and a small fraction (10\%) of the data. Then, the selection was applied to the full sample of data collected between 01.12.2004 and 31.12.2012. The number of events passing each of the selection criteria is 


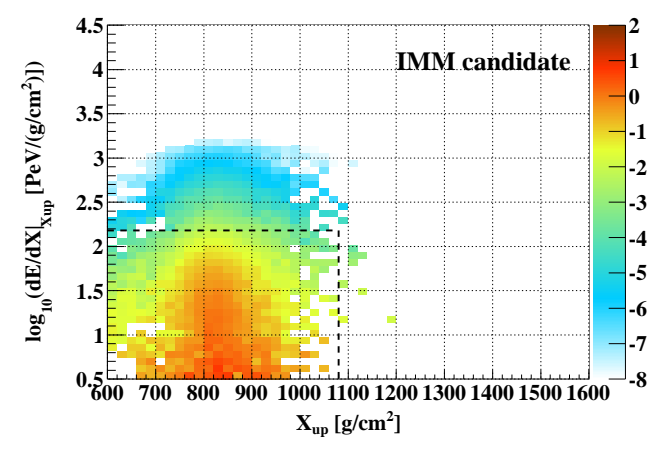

(a) Proton background

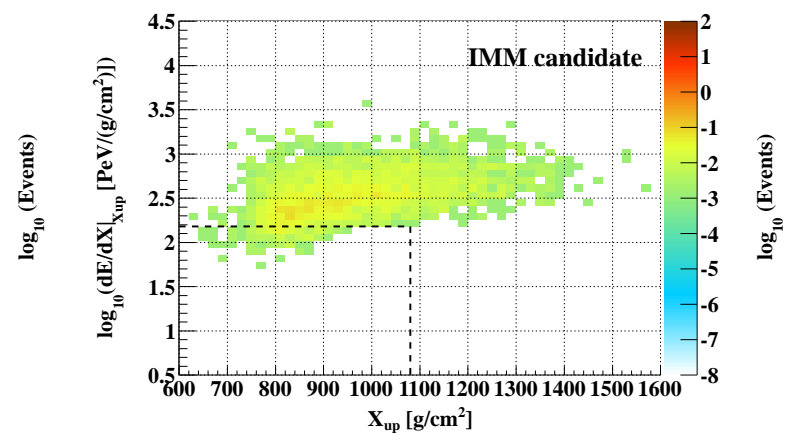

(b) Monopole signal, $\gamma=10^{11}$

Figure 2: (a) Correlation of $d E /\left.d X\right|_{X \text { up }}$ with $X_{\text {up }}$ for simulated UHECR proton showers passing the quality selection criteria. The color-coded scale indicates the number of events expected in the search period data set. Only events outside the dashed box are kept in the final selection for ultra-relativistic IMM. (b) Correlation of $d E /\left.d X\right|_{X \text { up }}$ with $X_{\text {up }}$ for simulated ultra-relativistic IMM of energy $10^{25} \mathrm{eV}$ with Lorentz factor $\gamma=10^{11}$. The color-coded scale indicates the number of events expected in the search period data set assuming a flux of $10^{-20}\left(\mathrm{~cm}^{2} \mathrm{sr} \mathrm{s}\right)^{-1}$. Only events outside the dashed box are kept in the final selection for ultra-relativistic IMM.

reported in Table $\mathbf{m}$. The correlation of $d E /\left.d X\right|_{X \text { up }}$ with $X_{\text {up }}$ for events passing the shower quality criteria and $X_{\max }>X_{\text {up }}$ is shown in Figure B(a).

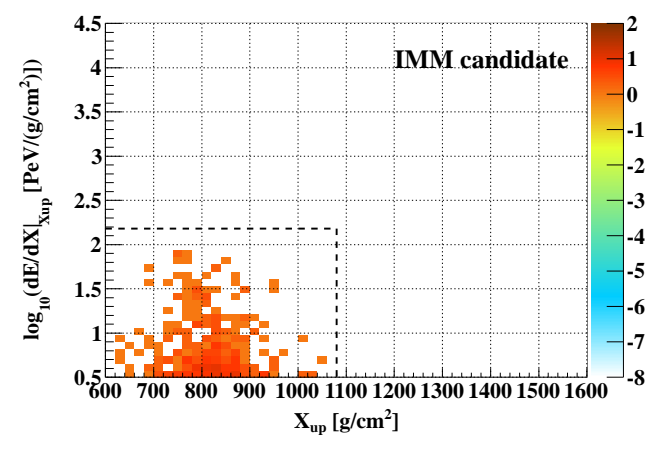

(a) Unblinded result

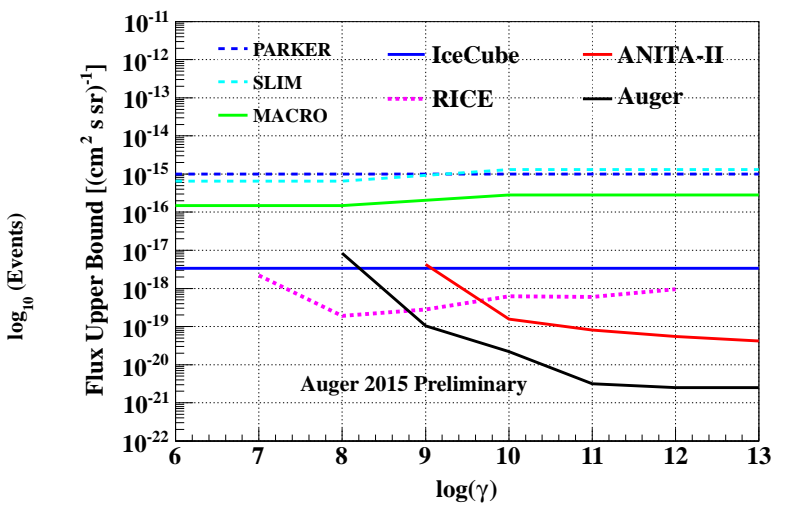

(b) $90 \%$ C.L. upper limit

Figure 3: (a) Correlation of $d E /\left.d X\right|_{X \text { up }}$ with $X_{\text {up }}$ for the data sample passing the shower quality selection criteria and $X_{\max }>X_{\text {up }}$. The color-coded scale indicates the number of events. No event is found outside the dashed box in the final selection for ultra-relativistic IMM. (b) $90 \%$ C.L. upper limits on the flux of ultra-relativistic IMM: this work (black solid line); Parker bound (blue dashed line) [ए]] ; SLIM (sky blue dashed line) [], MACRO (green solid line) [వ], IceCube (blue solid line) [ए]], RICE (pink dotted line) [Q] and ANITA-II (red line) [ए]]. The MACRO and SLIM limits above $\gamma=10^{9}$ were weakened by a factor of two to account for the IMM attenuation through Earth.

Given the null result of the search, a 90\% C.L. upper limit on the flux of ultra-relativistic IMM was derived. The flux $\Phi$ of ultra-relativistic IMM of Lorentz factor $\gamma$ is given by $\Phi(\gamma)=k / \mathscr{E}(\gamma)$ 
with a value $k=2.44$ (the Feldman-Cousins upper limit [20] for zero candidates and zero background events because the background events are conservatively assumed to be 0 instead of 0.07 expected events described in Section [1]). The exposure, $\mathscr{E}(\gamma)$, determined by Time Dependent Detector Simulation [ [2]], which makes use of slow control information and atmospheric measurements recorded during the data taking with considering the efficiency in IMM detection under the event selection criteria. The systematic uncertainty of the limit originating from the uncertainty of the exposure and $d E /\left.d X\right|_{X \text { up }}$ cut is $21 \%$. The propagation of uncertainties from the event statistics and systematics would lead to limit that is worse by a factor of 1.05 [22]. Here we follow a conservative approach and account for the systematics without propagation. We increase the limit by a factor $f=1+n \times 0.21$, where $n=1.28$ corresponds to $90 \%$ C.L. value. The corresponding upper limits for different Lorentz factors are shown in Figure B(b), together with results from previous experiments. Our result represents the best limit on ultra-relativistic IMM for $\gamma \geq 10^{9}$, with a factor of ten improvement with respect to previous experiments for $\gamma \geq 10^{10}$.

\section{Conclusions}

We have reported an analysis procedure and an event selection to search for signals produced by ultra-relativistic IMMs with FD of the Pierre Auger Observatory. Using this analysis, there is no candidate for IMMs signal in the data collected by the FD from 2004 to 2012. Therefore, we have evaluated a flux limit of $\Phi_{90 \% \text { C.L. }} \sim 10^{-20}\left(\mathrm{~cm}^{2} \mathrm{~s} \mathrm{sr}\right)^{-1}$ for $\log _{10}(\gamma) \geq 10$ which is a order of magnitude stronger than all previous published experimental limits.

\section{References}

[1] P. A. Dirac, Quantized Singularities in the Electromagnetic Field, Proc.Roy.Soc.Lond. A133 (1931) $60-72$.

[2] J. Preskill, Magnetic Monopoles, Ann.Rev.Nucl.Part.Sci. 34 (1984) 461-530.

[3] C. Escobar and R. Vazquez, Are high-energy cosmic rays magnetic monopoles?, Astropart.Phys. 10 (1999) 197-202, [astro-ph/9709148].

[4] S. D. Wick, T. W. Kephart, T. J. Weiler, and P. L. Biermann, Signatures for a cosmic flux of magnetic monopoles, Astropart.Phys. 18 (2003) 663-687, [astro-ph/0001233].

[5] MACRO Collaboration, M. Ambrosio et al., Final results of magnetic monopole searches with the MACRO experiment, Eur.Phys.J. C25 (2002) 511-522, [hep-ex/0207020].

[6] IceCube Collaboration, $\mathrm{H}$. Wissing, Search for relativistic magnetic monopoles with the AMANDA-II detector, Proc. of the 30th International Cosmic Ray Conference, Merida, Mexico (2007).

[7] Baikal Collaboration, V. Aynutdinov et al., Search for relativistic magnetic monopoles with the Baikal Neutrino Telescope NT200, Proc. of the 29th International Cosmic Ray Conference, Pune, India (2005) [astro-ph/050/713].

[8] S. Balestra, S. Cecchini, M. Cozzi, M. Errico, F. Fabbri, et al., Magnetic Monopole Search at high altitude with the SLIM experiment, Eur.Phys.J. C55 (2008) 57-63, [0801.4913].

[9] D. Hogan, D. Besson, J. Ralston, I. Kravchenko, and D. Seckel, Relativistic Magnetic Monopole Flux Constraints from RICE, Phys.Rev. D78 (2008) 075031, [0806.2129]. 
[10] ANITA-II Collaboration, M. Detrixhe et al., Ultra-Relativistic Magnetic Monopole Search with the ANITA-II Balloon-borne Radio Interferometer, Phys.Rev. D83 (2011) 023513, [1008.1282]].

[11] IceCube Collaboration, R. Abbasi et al., Search for Relativistic Magnetic Monopoles with IceCube, Phys.Rev. D87 (2013) 022001, [1208.486]].

[12] Pierre Auger Collaboration, A. Aab et al., The Pierre Auger Cosmic Ray Observatory, accepted for publication in Nucl.Instrum.Meth. A (2015) [1502.01323]].

[13] Pierre Auger Collaboration, I. Allekotte et al., The Surface Detector System of the Pierre Auger Observatory, Nucl.Instrum.Meth. A586 (2008) 409-420,

[14] Pierre Auger Collaboration, J. Abraham et al., The Fluorescence Detector of the Pierre Auger Observatory, Nucl.Instrum.Meth. A620 (2010) 227-251, [0907.4282]].

[15] S. Ahlen, Stopping Power Formula for Magnetic Monopoles, Phys.Rev. D17 (1978) 229-233.

[16] Pierre Auger Collaboration, A. Schulz, The measurement of the energy spectrum of cosmic rays above $3 \times 10^{17} \mathrm{eV}$ with the Pierre Auger Observatory, Proc. of the 33rd International Cosmic Ray Conference, Rio de Janeiro, Brazil (2013) [1507.5059].

[17] D. Heck, G. Schatz, T. Thouw, J. Knapp, and J. Capdevielle, CORSIKA: A Monte Carlo code to simulate extensive air showers, Forschungszentrum Karlsruhe Report FZKA (1998) 6019.

[18] S. Argiro, S. Barroso, J. Gonzalez, L. Nellen, T. C. Paul, et al., The Offline Software Framework of the Pierre Auger Observatory, Nucl.Instrum.Meth. A580 (2007) 1485-1496,

[19] E. N. Parker, The Origin of Magnetic Fields, Astrophys.J. 160 (1970) 383.

[20] G. J. Feldman and R. D. Cousins, A Unified approach to the classical statistical analysis of small signals, Phys.Rev. D57 (1998) 3873-3889, [physics/9711021].

[21] Pierre Auger Collaboration, P. Abreu et al., The exposure of the hybrid detector of the Pierre Auger Observatory, Astropart.Phys. 34 (2011) 368-381, [प0]0.6[62]].

[22] R. D. Cousins and V. L. Highland, Incorporating systematic uncertainties into an upper limit, Nucl.Instrum.Meth. A320 (1992) 331-335. 